\title{
Integral equation and simulation studies of a planar nematogenic liquid in the presence of a disorienting field
}

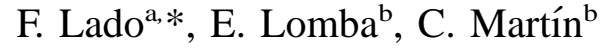 \\ ${ }^{a}$ Department of Physics, North Carolina State University, Raleigh, NC 27695-8202, USA \\ ' Instituto de Química Física Rocasolano, CSIC, Serrano 119, E-28006 Madrid, Spain
}

\begin{abstract}
Following a well-established method that uses integral equations for the study of anisotropic fluids, we analyze the behavior of a simple model of nematogens confined to plane in the presence of a disorienting field. Extensive computer simulation calculations support the validity of the integral equation results, which predict an isotropic-nematic transition at low temperatures and zero field and an in-plane order-disorder transition in the presence of a disorienting field at somewhat higher temperatures.
\end{abstract}

(C) 2003 Elsevier B.V. All rights reserved.

PACS: G1.20.Gy; 64.60.Cn; 64.70.Md

Keywords: Integral equations; Phase transitions; Nematogens; Order-disorder

\section{Introduction}

Biaxial nematics are a particular class of anisotropic materials characterized by the presence of two privileged directions. A typical example is offered by a system of coplanar molecules subject to a disorienting field perpendicular to the plane. This system is known to undergo an in-plane order-disorder transition at sufficiently low temperatures. The external field $\mathbf{W}_{0}$ and the director $\hat{\mathbf{n}}$ of the in-plane nematic phase define the two special directions [1,2]. This biaxiality modifies substantially the critical behavior of the orientational transition, which changes from first order [3] to second order in the limit of infinite disorienting field [4], being weakly first order for moderate fields.

Recently, Sokolovska, Sokolovskii, and Holovko ( $\mathrm{SSH}$ ) studied a simple model of a biaxial nematic in the presence of both an infinite disorienting field [4] i.e. an oriented plane rotator (OPR) model-and of a finite disorienting field [5]. In both the cases, the bulk systems were examined employing the anisotropic Ornstein-Zernike equation in the Mean Spherical Approximation, with Lovett's equation [6,7] used to link the one- and two-particle distribution functions. In this

\footnotetext{
*Corresponding author. Tel.: + 1-919-515-7050; fax: + 1-919-5156538 .

E-mail address: fred_lado@ncsu.edu (F. Lado).
}

article, we study the same biaxial nematic model in a finite disorienting field, as in Ref. [5], but with the molecular centers now constrained to lie in a plane. We exploit a related integral equation approach that has been successfully used to describe the ferromagnetic transition in bulk Heisenberg spin fluids $[9,10]$ as well as the behavior of a planar dipolar system [11]. As in Ref. [5], the core of the treatment is the OrnsteinZernike (OZ) equation for anisotropic fluids, but here supplemented with the first member of the KirkwoodBorn-Green-Yvon (KBGY) hierarchy [8] to link the one- and two-particle distribution functions. Specific to the treatment used in Refs. [9-11] and in this work is the technique of expanding distribution functions in terms of generalized spherical harmonics constructed with the single particle density as weight function, which simplifies the resulting formalism.

This study of a biaxial nematic model in a plane, which is dedicated to Prof. Myroslav Holovko on the occasion of his 60th birthday, is carried out using both the reference hypernetted chain (RHNC) approximation and computer simulation. The latter makes possible an assessment of the quality of our theoretical description.

The rest of the article is organized as follows. The theory and details of the model are presented in Section 2 , while expressions for the thermodynamics and relevant structural quantities are collected in Section 3. 
Finally, we present in Section 4 our most significant results and conclusions.

\section{Formulation of the integral equations}

\subsection{Nematogens in a plane}

Following SSH [5], we write the total potential energy of a configuration of $N$ molecules whose centers of mass are constrained to lie in a plane of area $A$ as

$U=\sum_{j} u_{\mathrm{ext}}\left(\omega_{j}\right)+\sum_{i<j} u_{\mathrm{HS}}\left(r_{i j}\right)+\sum_{i<j} u\left(r_{i j}, \omega_{i}, \omega_{j}\right)$,

where $\omega=(\theta, \phi)$ is the orientation of a molecular nematic axis, which is free to rotate in three-dimensions, referred to the external field direction as the $z$-axis perpendicular to the plane of $A$. The energies here are the external disorienting potential,

$\beta u_{\text {ext }}(\omega)=W_{0} P_{2}(\cos \theta)$, with $W_{0}>0$,

the hard sphere potential, $u_{\mathrm{HS}}(r)$, for spheres of diameter $\sigma$, and an attractive potential between a pair of molecules that tends to align their nematic axes,

$u\left(r, \omega_{1}, \omega_{2}\right)=-u_{0}(r) P_{2}\left(\cos \theta_{12}\right)$,

$\beta u_{0}(r)=K \frac{\mathrm{e}^{-\kappa(r / \sigma-1)}}{r / \sigma}, \quad r>\sigma$.

In these expressions, $P_{2}(x)$ is the Legendre polynomial of the order two, $\theta_{12}$ the angle between the axes of molecules 1 and $2, \kappa$ a dimensionless range parameter, and $\beta=\left(k_{\mathrm{B}} T\right)^{-1}$ the inverse temperature, with $k_{\mathrm{B}}$ Boltzmann's constant. In the calculation, the dimensionless coupling strength $K$ will serve as the reduced inverse temperature, $K=1 / T^{*}$. The external disorienting field [Eq. (2)] is the first non-vanishing term in an expansion in Legendre polynomials of some more general external potential. It can be viewed as a simple-minded representation of the interaction between an external electric field and a molecule with its dipole perpendicular to its long axis [3]. Thus, the molecules tend to lie perpendicular to the external field.

The canonical partition function

$Z=\frac{1}{N !\left(4 \pi \Lambda^{2}\right)^{N}} \int \prod_{j=1}^{N}\left(\mathrm{~d} \mathbf{r}_{j} \mathrm{~d} \omega_{j}\right) \mathrm{e}^{-\beta U}$

can be factored into an ideal part and the excess, $Z=$ $Z^{\text {id }} Z^{\text {ex }}$, where

$$
\begin{aligned}
Z^{\mathrm{id}}= & \frac{1}{N !\left(4 \pi \Lambda^{2}\right)^{N}} \prod_{j=1}^{N}\left(\mathrm{~d} \mathbf{r}_{j} \mathrm{~d} \omega_{j}\right) \\
& \times \exp \left[-\beta \sum_{j} u_{\mathrm{ext}}\left(\omega_{j}\right)\right] \\
= & \frac{1}{N !}\left(\frac{A}{\Lambda^{2}} \frac{\operatorname{erf}\left[\left(3 W_{0} / 2\right)^{1 / 2}\right] \mathrm{e}^{W_{0} / 2}}{2\left(3 W_{0} / 2 \pi\right)^{1 / 2}}\right)^{N}, \\
Z^{\mathrm{ex}}= & \frac{1}{(4 \pi A)^{N}} \int_{j=1}^{N}\left[\mathrm{~d} \mathbf{r}_{j} \mathrm{~d} \omega_{j} f_{0}\left(\omega_{j}\right)\right] \\
& \times \exp \left(-\beta \sum_{i<j}\left[u_{\mathrm{HS}}\left(r_{i j}\right)+u\left(r_{i j}, \omega_{i}, \omega_{j}\right)\right]\right) .
\end{aligned}
$$

Here,

$f_{0}(\omega)=\frac{2\left(3 W_{0} / 2 \pi\right)^{1 / 2}}{\operatorname{erf}\left[\left(3 W_{0} / 2\right)^{1 / 2}\right]} \exp \left(-\frac{3}{2} W_{0} \cos ^{2} \theta\right)$

is an angular distribution function of the molecular axes in the external field for non-interacting molecules, with $\operatorname{erf}(x)$ the error function, and we have conventionally introduced above the de Broglie thermal wavelength $\Lambda$ for the normalization of $Z$. The distribution function $f_{0}(\omega)$ is normalized so that

$\frac{1}{4 \pi} \int \mathrm{d} \omega f_{0}(\omega)=1$

\subsection{One-body and two-body distribution functions}

With the molecules subject to both one-body and two-body interactions, a statistical description of the system requires both the one-body and two-body density functions,

$$
\begin{gathered}
\rho^{(1)}(\mathbf{r}, \omega)=\left\langle\sum_{j=1}^{N} \delta\left(\mathbf{r}-\mathbf{r}_{j}\right) \delta\left(\omega-\omega_{j}\right)\right\rangle=\frac{\rho}{4 \pi} f(\omega), \\
\rho^{(2)}\left(\mathbf{r}, \omega, \mathbf{r}^{\prime}, \omega^{\prime}\right)=\left\langle\sum_{i \neq j} \delta\left(\mathbf{r}-\mathbf{r}_{i}\right) \delta\left(\omega-\omega_{i}\right)\right. \\
\left.\quad \times \delta\left(\mathbf{r}^{\prime}-\mathbf{r}_{j}\right) \delta\left(\omega^{\prime}-\omega_{j}\right)\right\rangle \\
=\frac{\rho^{2}}{(4 \pi)^{2}} f(\omega) f\left(\omega^{\prime}\right) g\left(\left|\mathbf{r}-\mathbf{r}^{\prime}\right|, \omega, \omega^{\prime}\right),
\end{gathered}
$$

where $\rho=N / A$ is the planar density and $f(\omega)$ is the one-body angular distribution function in the interacting 
fluid. Eq. (11) defines the generalized pair distribution function $g\left(r, \omega, \omega^{\prime}\right)$ of the anisotropic system. The angular brackets in these definitions denote a canonical ensemble average with the Boltzmann factor of Eq. (5) and the potential Eq. (1).

The basic equations that determine the distribution functions $f(\omega)$ and $g\left(r, \omega, \omega^{\prime}\right)$ are well known [12,13]. The one-body density can be differentiated with respect to $x=\cos \theta$ to give

$$
\begin{aligned}
& \frac{\mathrm{d}}{\mathrm{d} x_{1}} \ln \left[\frac{f\left(\omega_{1}\right)}{f_{0}\left(\omega_{1}\right)}\right]= \\
& \quad-\frac{\rho}{4 \pi} \int \mathrm{d} \mathbf{r}_{2} \mathrm{~d} \omega_{2} f\left(\omega_{2}\right) g\left(r_{12}, \omega_{1}, \omega_{2}\right) \frac{\mathrm{d} \beta u\left(r_{12}, \omega_{1}, \omega_{2}\right)}{\mathrm{d} x_{1}},
\end{aligned}
$$

the first member of a KBGY hierarchy. Calculation of $f(\omega)$ from this equation requires knowing $g\left(r, \omega_{1}, \omega_{2}\right)$. In classical liquid state theory, the pair distribution function is obtained from the Ornstein-Zernike equation and a closure relation $[12,13]$. The first of these, generalized for anisotropy, reads

$$
\begin{aligned}
\gamma\left(r_{12}, \omega_{1}, \omega_{2}\right)= & \frac{\rho}{4 \pi} \int \mathrm{d} \mathbf{r}_{3} \mathrm{~d} \omega_{3} f\left(\omega_{3}\right)\left[\gamma\left(r_{13}, \omega_{1}, \omega_{3}\right)\right. \\
& \left.+c\left(r_{13}, \omega_{1}, \omega_{3}\right)\right] c\left(r_{32}, \omega_{3}, \omega_{2}\right)
\end{aligned}
$$

for the indirect correlation function $\gamma=g-1-c$, where $c\left(r, \omega_{1}, \omega_{2}\right)$ is the direct correlation function. The second, or closure, relation expresses $c\left(r, \omega_{1}, \omega_{2}\right)$ back in terms of $\gamma\left(r, \omega_{1}, \omega_{2}\right)$ and the model's pair interactions,

$$
\begin{aligned}
c\left(r, \omega_{1}, \omega_{2}\right)= & \exp \left[-\beta u_{\mathrm{HS}}(r)-\beta u\left(r, \omega_{1}, \omega_{2}\right)\right. \\
& \left.+\gamma\left(r, \omega_{1}, \omega_{2}\right)+b\left(r, \omega_{1}, \omega_{2}\right)\right]-1 \\
& -\gamma\left(r, \omega_{1}, \omega_{2}\right) .
\end{aligned}
$$

This relation must be supplemented with an approximation for $b\left(r, \omega_{1}, \omega_{2}\right)$, the so-called bridge function, which is formally defined in terms of a diagram summation [12] that offers little practical benefit. Most approximate closures for $c\left(r, \omega_{1}, \omega_{2}\right)$ define $b\left(r, \omega_{1}, \omega_{2}\right)$ implicitly. We note again that the derivative of $f(\omega)$ in Eq. (12) can alternatively be related to the direct correlation function $c\left(r, \omega_{1}, \omega_{2}\right)$ through the LovettMou-Buff-Wertheim equation [6,7], which was the route chosen by $\mathrm{SSH}[4,5]$.

\subsection{Generalized spherical harmonics}

As with the case of isotropic molecular fluids [14], Eqs. (12)-(14) can be made tractable by expansion of all angle-dependent functions, such as $\ln f(\omega)$ and $\gamma\left(r, \omega_{1}, \omega_{2}\right)$, in standard spherical harmonics $Y_{l m}(\omega)$, as exemplified in a number of recent works [5,15-17]. This leads, however, to the appearance in the resulting equations of new matrix elements of the general form

$A_{m_{1} m_{2}}^{l_{1} l_{2}}=\int \mathrm{d} \omega f(\omega) Y_{l_{1} m_{1}}(\omega) Y_{l_{2} m_{2}}^{*}(\omega)$

that do not appear (because of orthogonality) in the similar formalism for isotropic molecular fluids, where $f(\omega)=1$. To avoid this extra complication, here we will instead expand in generalized spherical harmonics $\mathscr{F}_{I m}(\omega)$ that are explicitly constructed to be orthogonal with weight function $f(\omega)$,

$\int \mathrm{d} \omega f(\omega) \mathscr{Y}_{l_{1} m_{1}}(\omega) \mathscr{Y}_{l_{2} m_{2}}^{*}(\omega)=\delta_{l_{1} l_{2}} \delta_{m_{1} m_{2}}$,

so that the resulting formalism is then identical to that of isotropic molecular fluids $[10,11]$. Since the anisotropy of the present model affects only the polar angle $\theta$, this entails constructing just the generalized Legendre functions $\mathscr{P}_{l m}(\cos \theta)$, which we normalize so that

$\frac{1}{2} \int_{-1}^{1} \mathrm{~d} x f(x) \mathscr{P}_{l m}(x) \mathscr{P}_{l^{\prime} m}(x)=\delta_{l l^{\prime}}$

The generalized spherical harmonics are then

$\mathscr{Y}_{l m}(\omega)=\frac{1}{\sqrt{4 \pi}}(-1)^{m} \mathrm{e}^{i m \phi} \mathscr{P}_{l m}(\cos \theta)$,

and pair functions such as $\gamma\left(r, \omega_{1}, \omega_{2}\right)$ are written out as

$$
\gamma\left(r, \omega_{1}, \omega_{2}\right)=4 \pi \sum_{l_{1}, l_{2}, m} \gamma_{l_{1} l_{2} m}(r) \mathscr{Y} l_{1} m\left(\omega_{1}\right) \mathscr{Y}_{l_{2} \bar{m}}\left(\omega_{2}\right),
$$

where $\bar{m}=-m$. Because the potentials in Eq. (1) are invariant to a $180^{\circ}$ flip of a molecular axis, indices $l_{1}$ and $l_{2}$ will here be even integers; further, the expansion coefficients satisfy the general symmetries $\gamma_{l_{2} l_{1} m}(r)=$ $\gamma_{l_{1} l_{2} m}(r)$ and $\gamma_{l_{1} l_{2} \bar{m}}(r)=\gamma_{l_{1} l_{2} m}(r)$. For the pair potential $u\left(r, \omega_{1}, \omega_{2}\right)$ of Eq. (3) we get explicitly

$u_{l_{1} l_{2} m}(r)=-u_{0}(r) P_{l_{1} l_{2} m}$,

with non-zero elements

$P_{000}=\frac{1}{4}\left(3\left\langle x^{2}\right\rangle-1\right)^{2}$

$P_{200}=P_{020}=\frac{3}{4}\left(3\left\langle x^{2}\right\rangle-1\right)\left(\left\langle x^{4}\right\rangle-\left\langle x^{2}\right\rangle^{2}\right)^{1 / 2}$, 
$P_{220}=\frac{9}{4}\left(\left\langle x^{4}\right\rangle-\left\langle x^{2}\right\rangle^{2}\right)$

$P_{221}=P_{22-1}=-\frac{3}{2}\left\langle x^{2}\left(1-x^{2}\right)\right\rangle$,

$P_{222}=P_{22-2}=\frac{3}{8}\left\langle\left(1-x^{2}\right)^{2}\right\rangle$,

where $\left\langle x^{k}\right\rangle$ is the $k$ th moment of $f(x)$. In the absence of an external field $\left(W_{0}=0\right)$, we have $\left\langle x^{2}\right\rangle=1 / 3,\left\langle x^{4}\right\rangle=$ $1 / 5$ and so $P_{000}=P_{200}=0, P_{220}=-P_{221}=P_{222}=1 / 5$.

\subsection{OZ equation and the RHNC closure}

The OZ Eq. (13) is more conveniently used after deconvolution by Fourier transformation, which yields

$$
\begin{aligned}
\tilde{\gamma}\left(k, \omega_{1}, \omega_{2}\right)= & \frac{\rho}{4 \pi} \int \mathrm{d} \omega_{3} f\left(\omega_{3}\right)\left[\tilde{\gamma}\left(k, \omega_{1}, \omega_{3}\right)\right. \\
& \left.+\tilde{c}\left(k, \omega_{1}, \omega_{3}\right)\right] \tilde{c}\left(k, \omega_{3}, \omega_{2}\right),
\end{aligned}
$$

with a final integration still remaining. Because the orientations of the molecular axes $\omega_{1}, \omega_{2}$ and that of $\mathbf{r}_{12}$ are decoupled in the present model, the transforms may be performed holding the former fixed. In twodimensions, the Fourier transform (FT) of a circularly symmetric function becomes a Hankel transform, and so we get a transform pair

$\tilde{\gamma}\left(k, \omega_{1}, \omega_{2}\right)=2 \pi \int_{0}^{\infty} \mathrm{d} r r \gamma\left(r, \omega_{1}, \omega_{2}\right) J_{0}(k r)$,

$\gamma\left(r, \omega_{1}, \omega_{2}\right)=\frac{1}{2 \pi} \int_{0}^{\infty} \mathrm{d} k k \tilde{\gamma}\left(k, \omega_{1}, \omega_{2}\right) J_{0}(k r)$,

where $J_{0}(x)$ is the Bessel function of the order zero. The transformed functions are then expanded just as in Eq. (19),

$\tilde{\gamma}\left(k, \omega_{1}, \omega_{2}\right)=4 \pi \sum_{l_{1}, l_{2}, m} \tilde{\gamma}_{l_{1} l_{2} m}(k) \mathscr{Y} l_{1} m\left(\omega_{1}\right) \mathscr{Y} l_{2} \bar{m}\left(\omega_{2}\right)$,

and the final integration in Eq. (26) can be carried out to give matrix equations,

$\tilde{\gamma}_{l_{1} l_{2} m}(k)=(-1)^{m} \rho \sum_{l_{3}}\left[\tilde{\gamma}_{l_{1} l_{3} m}(k)+\tilde{c}_{l_{1} l_{3} m}(k)\right] \tilde{c}_{l_{3} l_{2} m}(k)$

that are solved by matrix operations for the $\tilde{\gamma}_{l_{1} l_{2} m}(k)$ in terms of the $\tilde{c}_{l_{1} l_{2} m}(k)$. One cycle of the iterative (numerical) solution that yields a converged set of coefficients $\left\{\gamma_{l_{1} l_{2} m}(r)\right\}$ will then consist of four steps:

$$
\begin{aligned}
& \left\{\gamma_{l_{1} l_{2} m}(r)\right\}^{\text {closure }} \rightarrow\left\{c_{l_{1} l_{2} m}(r)\right\} \stackrel{\text { (FT) }}{\rightarrow}\left\{\tilde{c}_{l_{1} l_{2} m} \stackrel{\text { OZ }}{\rightarrow}\left\{\tilde{\gamma}_{l_{1} l_{2} m}(k)\right\}\right. \\
& \stackrel{(\mathrm{FT})^{-1}}{\rightarrow}\left\{\gamma_{l_{1} l_{2} m}(r)\right\} .
\end{aligned}
$$

The specific closure relation used in this work is the RHNC equation [18], in which the needed bridge function $b\left(r, \omega_{1}, \omega_{2}\right)$ is approximated by the known bridge function of some selected reference system, generally a hard-core model. For the two-dimensional fluid treated here, we take as reference system a hard disk (HD) fluid at the same density, and put

$b\left(r, \omega_{1}, \omega_{2}\right) \approx b_{\mathrm{HD}}(r)$.

There is no parameterized solution known for the hard disk fluid that maintains internal thermodynamic consistency, and so to get $b_{\mathrm{HD}}(r)$ we resort to an approximate closure for this model that achieves at least pressure consistency [19], namely

$$
\begin{aligned}
c_{\mathrm{HD}}(r)= & h_{\mathrm{HD}}(r)-(1-\mu)\left[g_{\mathrm{HD}}(r) \mathrm{e}^{\beta u_{\mathrm{HD}}(r)}-1\right] \\
& -\mu \ln \left[g_{\mathrm{HD}}(r) \mathrm{e}^{\beta u_{\mathrm{HD}}(r)}\right] .
\end{aligned}
$$

This is a blend of the familiar Percus-Yevick $(\mu=$ $0)$ and hypernetted-chain $(\mu=1)$ closures controlled by the parameter $\mu$ to achieve consistency of the virial and compressibility pressures. The density dependence of $\mu$ constrained in this way is found by calculation to be fitted by

$$
\begin{aligned}
\mu= & 0.0920+0.1222\left(\rho \sigma^{2}\right) \\
& +0.1642\left(\rho \sigma^{2}\right)^{2}+0.1100\left(\rho \sigma^{2}\right)^{3} .
\end{aligned}
$$

A better approximation for $b\left(r, \omega_{1}, \omega_{2}\right)$ would include anisotropy with at least a $P_{2}\left(\cos \theta_{12}\right)$ term, as in the pair potential, but essentially nothing is known about such generalized bridge functions.

The Hankel transforms needed in these calculations must be evaluated numerically. The discrete versions $[19,20]$ of the integrals Eqs. (27) and (28) used in this work,

$\tilde{f}\left(k_{j}\right)=\frac{4 \pi}{K^{2}} \sum_{i=1}^{N_{r}-1} f\left(r_{i}\right) \frac{J_{0}\left(k_{j} r_{i}\right)}{J_{1}^{2}\left(K r_{i}\right)}$,

$f\left(r_{i}\right)=\frac{1}{\pi R^{2}} \sum_{j=1}^{N_{r}-1} \tilde{f}\left(k_{j}\right) \frac{J_{0}\left(k_{j} r_{i}\right)}{J_{1}^{2}\left(k_{j} R\right)}$,

preserve the orthogonality of the continuous Hankel 
transforms. In these expressions, $J_{n}(x)$ is the Bessel function of the order $n$ and the numerical grids are defined by $r_{i}=\lambda_{i} / K, k_{j}=\lambda_{j} / R$, where $\lambda_{j}$ is the $j$ th root of $J_{0}(x)$ and $K \equiv k_{N_{r}}, R \equiv r_{N_{r}}$; the range $R$ and number of grid points $N_{r}$ are free choices.

\subsection{KBGY equation for $f(x)$}

The iterative solution for $\gamma\left(r_{12}, \omega_{1}, \omega_{2}\right)$ described in the previous section depends on the current form of the angular distribution function $f(x)$, as reflected in the pair potential coefficients $P_{l_{1} l_{2} m}$, Eqs. (21)-(25); now $f(x)$ must be updated through Eq. (12) using the new pair function

$$
\begin{array}{r}
g\left(r, \omega_{1}, \omega_{2}\right)=\exp \left[-\beta u_{\mathrm{HS}}(r)-\beta u\left(r, \omega_{1}, \omega_{2}\right)\right. \\
\left.+\gamma\left(r, \omega_{1}, \omega_{2}\right)+b\left(r, \omega_{1}, \omega_{2}\right)\right]
\end{array}
$$

and the pair potential $u\left(r_{12}, \omega_{1}, \omega_{2}\right)$. Expanding these functions in the generalized spherical harmonics, we get for the KBGY Eq. (12),

$$
\frac{\mathrm{d}}{\mathrm{d} x} \ln \left[\frac{f(x)}{f_{0}(x)}\right]=-\sum_{l_{1}, l_{2}, m} \xi_{l_{1} l_{2} m} \mathscr{P}_{l_{1} m}(x) \frac{\mathrm{d} \mathscr{P}_{l_{2} m}(x)}{\mathrm{d} x},
$$

where

$\xi_{l_{1} l_{2} m}=\rho \int \mathrm{d} \mathbf{r} \sum_{l_{3}} g_{l_{1} l_{3} m}(r) \beta u_{l_{3} l_{2} m}(r)$

Only the coefficients $\xi_{020}, \xi_{220}, \xi_{221}$, and $\xi_{222}$ contribute and we get

$$
\begin{aligned}
\ln f(x)= & \ln f_{0}(x)-F(x)+\text { const, } \\
F(x) \equiv & \xi_{020} \mathscr{P}_{20}(x) \\
& +\frac{1}{2} \xi_{220} \mathscr{P}_{20}^{2}(x)+\xi_{221} \mathscr{P}_{21}^{2}(x)+\xi_{222} \mathscr{P}_{22}^{2}(x) .
\end{aligned}
$$

The new distribution function $f(x)$ is finally found as

$$
\ln f(x)=\ln f_{0}(x)-\sum_{l \geq 1} a_{l} \mathscr{P}_{l 0}(x)+\text { const },
$$

$a_{l} \equiv \frac{1}{2} \int_{-1}^{1} \mathrm{~d} x f(x) F(x) \mathscr{P}_{l 0}(x)$

Odd coefficients $a_{1}, a_{3}, \ldots$ vanish and, in practice, we find that the coefficient $a_{2}$ is at least two orders of magnitude larger than $a_{4}$, so that $f(x)$ remains a Gaussian, $\ln f(x)=-\frac{3}{2} W x^{2}+$ const,

with an interaction-altered field strength

$W=W_{0}+\frac{2 a_{2}}{3\left[\left\langle x^{4}\right\rangle-\left\langle x^{2}\right\rangle^{2}\right]^{1 / 2}}$.

The constant in Eq. (44) is determined by normalization (see Eq. (8)).

The integrals Eq. (43) are evaluated numerically and exactly using Gaussian quadrature,

$a_{l}=\sum_{j=1}^{n} w\left(x_{j}\right) F\left(x_{j}\right) \mathscr{P}_{l 0}\left(x_{j}\right)$

with $l<n$, where the $x_{j}$ are the $n$ zeroes of $\mathscr{P}_{n 0}(x)$ and $w\left(x_{j}\right)$ are the corresponding Gaussian weights. These quantities, along with the generalized Legendre functions themselves, are calculated using a robust algorithm proposed by Press and Teukolsky [21].

Successive rounds of iterations for $\gamma\left(r_{12}, \omega_{1}, \omega_{2}\right)$ and $f(x)$ are repeated until both functions are self-consistently converged.

\section{Thermodynamic and structural quantities}

Once the one- and two-particle distribution functions have been determined, it is straightforward to calculate the various thermodynamic quantities. For instance, the excess internal energy $\beta U / N$ and the compressibility factor $\beta p / \rho$ are found as quadratures,

$$
\begin{aligned}
\frac{\beta U}{N}= & \frac{1}{2} \rho \int \mathrm{d} \mathbf{r} \sum_{l_{1}, l_{2}, m} g_{l_{1} l_{2} m}(r) \beta u_{l_{1} l_{2} m}(r), \\
\frac{\beta p}{\rho}= & 1+\frac{1}{2} \pi \rho \sigma^{2} g_{000}(\sigma) \\
& -\frac{1}{4} \rho \int \mathrm{d} \mathbf{r} \sum_{l_{1}, l_{2}, m} g_{l_{1} l_{2} m}(r) r \frac{\mathrm{d} \beta u_{l_{1} l_{2} m}(r)}{\mathrm{d} r},
\end{aligned}
$$

in terms of the coefficients of the expansion of the pair distribution function and potential function in the generalized spherical harmonics.

An important quantity is the susceptibility, which measures the response of the system to an external field. If one assumes the external field to be of the general form $A_{x}(\sin \theta \cos \phi)^{2}+A_{y}(\sin \theta \sin \phi)^{2}+A_{z}(\cos \theta)^{2}$, then the response of the system will be given by the following 'polarization,'

$M_{x}=\frac{1}{\beta} \frac{\partial \ln Z}{\partial A_{x}}=\left\langle(\sin \theta)^{2}\right\rangle\left\langle(\cos \phi)^{2}\right\rangle=\frac{1}{2}\left(1-\left\langle x^{2}\right\rangle\right)$, 
$M_{y}=\frac{1}{\beta} \frac{\partial \ln Z}{\partial A_{y}}=\left\langle(\sin \theta)^{2}\right\rangle\left\langle(\sin \phi)^{2}\right\rangle=\frac{1}{2}\left(1-\left\langle x^{2}\right\rangle\right)$,

$M_{z}=\frac{1}{\beta} \frac{\partial \ln Z}{\partial A_{z}}=\left\langle(\cos \theta)^{2}\right\rangle=\left\langle x^{2}\right\rangle$,

where the $\phi$ averages are taken at zero $\mathbf{A}$ field. The susceptibility tensor is then defined by

$\chi_{\alpha \beta}=\frac{1}{N} \frac{\partial M_{\alpha}}{\partial A_{\beta}}$

at zero A field, or more explicitly

$\chi=\left(\begin{array}{lll}a+d & a-d & b \\ a-d & a+d & b \\ b & b & c\end{array}\right)$

where

$$
\begin{aligned}
a= & \frac{1}{4}\left(1-\left\langle x^{2}\right\rangle\right)^{2}\left[1+\rho \tilde{h}_{000}(0)\right]-\frac{1}{4}\left(1-\left\langle x^{2}\right\rangle\right) \\
& \times\left(\left\langle x^{4}\right\rangle-\left\langle x^{2}\right\rangle^{2}\right)^{1 / 2}\left[\rho \tilde{h}_{200}(0)+\rho \tilde{h}_{020}(0)\right] \\
& +\frac{1}{4}\left(\left\langle x^{4}\right\rangle-\left\langle x^{2}\right\rangle^{2}\right)\left[1+\rho \tilde{h}_{220}(0)\right],
\end{aligned}
$$$$
b=\frac{1}{2}\left\langle x^{2}\right\rangle\left(1-\left\langle x^{2}\right\rangle\right)\left[1+\rho \tilde{h}_{000}(0)\right]+\frac{1}{4}\left(1-2\left\langle x^{2}\right\rangle\right)
$$$$
\left(\left\langle x^{4}\right\rangle-\left\langle x^{2}\right\rangle^{2}\right)^{1 / 2}\left[\rho \tilde{h}_{200}(0)+\rho \tilde{h}_{020}(0)\right]
$$$$
-\frac{1}{2}\left(\left\langle x^{4}\right\rangle-\left\langle x^{2}\right\rangle^{2}\right)\left[1+\rho \tilde{h}_{220}(0)\right]
$$$$
c=\left\langle x^{2}\right\rangle^{2}\left[1+\rho \tilde{h}_{000}(0)\right]
$$$$
+\left\langle x^{2}\right\rangle\left(\left\langle x^{4}\right\rangle-\left\langle x^{2}\right\rangle^{2}\right)^{1 / 2}\left[\rho \tilde{h}_{200}(0)+\rho \tilde{h}_{020}(0)\right]
$$$$
+\left(\left\langle x^{4}\right\rangle-\left\langle x^{2}\right\rangle^{2}\right)\left[1+\rho \tilde{h}_{220}(0)\right]
$$$$
d=\frac{1}{8}\left\langle\left(1-x^{2}\right)^{2}\right\rangle\left[1+\rho \tilde{h}_{222}(0)\right] .
$$

These combinations satisfy $4(a+b)+c=1+\rho \tilde{h}_{000}(0)$ Note that those components $\chi_{\alpha \beta}$ that involve the $z$-axis do not depend on $\tilde{h}_{222}(0)$.

From all the expansion coefficients of the pair distri- bution function, two deserve special attention, namely, the center-to-center distribution function, $g_{000}(r)$, and the shell average, $\left\langle\frac{1}{2}\left(3 \cos ^{2} \theta_{12}-1\right)\right\rangle(r)$, which is essentially given by

$$
\begin{aligned}
\rho^{220}(r) / \rho^{2} & \equiv 5\left\langle\rho^{(2)}(12) P\left(\cos \theta_{12}\right)\right\rangle_{\omega_{1} \omega_{2}} / \rho^{2} \\
& =5 \sum_{l_{1}, l_{2}, m} g_{l_{1} l_{2} m}(r) P_{l_{1} l_{2} m} .
\end{aligned}
$$

In the limit of zero field $\left(W_{0}=0\right)$, this becomes the usual spherical harmonic coefficient

$$
g^{220}(r)=g_{220}(r)-2 g_{221}(r)+2 g_{222}(r) .
$$

This quantity is a measure of the average relative orientation between two fluid particles.

\section{Results}

We have considered the systems with parameters $\kappa=$ 1.0 and 0.5 , reduced densities $\rho \sigma^{2}=0.6$ and 0.8 , and

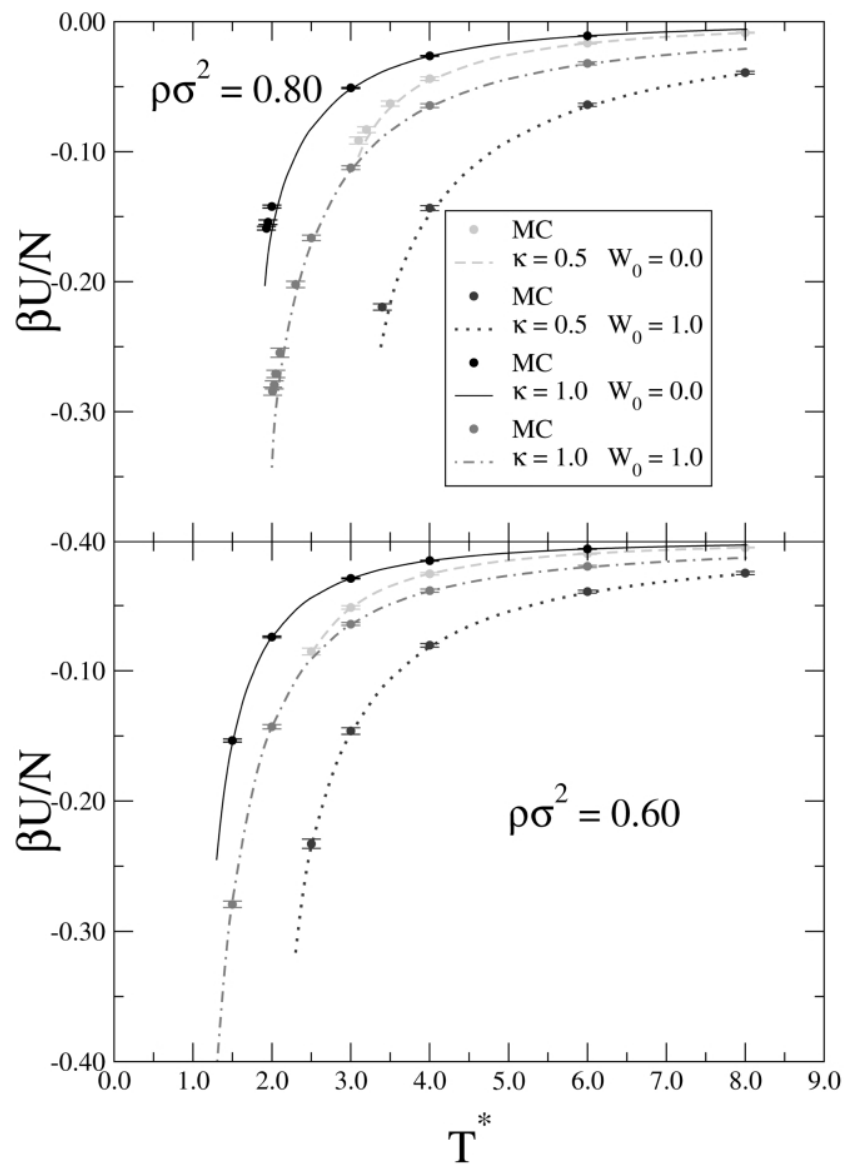

Fig. 1. Excess internal energy for the biaxial nematic fluid in a plane with and without external field for the states studied in this work. Lines denote RHNC results and symbols correspond to MC values. 
Table 1

Effective field $W$, compressibility factor $\beta p / \rho$, and Fourier transforms at $k=0$ of three coefficients of the orthogonal polynomial expansion of the total correlation function $h=g-1$, for a biaxial nematic model at $\rho \sigma^{2}=0.6$

\begin{tabular}{|c|c|c|c|c|c|c|c|c|}
\hline \multirow[t]{2}{*}{$T^{*}$} & \multirow[t]{2}{*}{$\kappa$} & \multirow[t]{2}{*}{$W_{0}$} & \multirow[t]{2}{*}{$W$} & \multicolumn{2}{|l|}{$\beta p / \rho$} & \multirow[t]{2}{*}{$\rho \tilde{h}_{000}(0)$} & \multirow[t]{2}{*}{$\rho \tilde{h}_{220}(0)$} & \multirow[t]{2}{*}{$\rho \tilde{h}_{222}(0)$} \\
\hline & & & & RHNC & $\mathrm{MC}$ & & & \\
\hline 8.00 & 0.5 & 0 & 0.000 & 3.631 & $3.652(49)$ & -0.9010 & 0.269 & 0.269 \\
\hline 6.00 & 0.5 & 0 & 0.000 & 3.628 & $3.656(45)$ & -0.9009 & 0.394 & 0.394 \\
\hline 4.00 & 0.5 & 0 & 0.000 & 3.616 & $3.641(45)$ & -0.9006 & 0.739 & 0.739 \\
\hline 3.00 & 0.5 & 0 & 0.000 & 3.595 & $3.626(52)$ & -0.9001 & 1.322 & 1.322 \\
\hline 2.50 & 0.5 & 0 & 0.000 & 3.566 & $3.596(40)$ & -0.8991 & 2.235 & 2.234 \\
\hline 2.15 & 0.5 & 0 & -0.007 & 3.502 & & -0.8954 & 5.958 & 5.885 \\
\hline 6.00 & 1.0 & 0 & 0.000 & 3.630 & $3.659(22)$ & -0.9010 & 0.186 & 0.186 \\
\hline 4.00 & 1.0 & 0 & 0.000 & 3.622 & $3.651(21)$ & -0.9008 & 0.307 & 0.307 \\
\hline 3.00 & 1.0 & 0 & 0.000 & 3.610 & $3.634(22)$ & -0.9005 & 0.456 & 0.456 \\
\hline 2.00 & 1.0 & 0 & 0.000 & 3.570 & $3.599(22)$ & -0.8996 & 0.889 & 0.889 \\
\hline 1.50 & 1.0 & 0 & -0.002 & 3.493 & $3.540(25)$ & -0.8974 & 1.743 & 1.741 \\
\hline 1.27 & 1.0 & 0 & -0.017 & 3.372 & & -0.8918 & 4.038 & 3.974 \\
\hline 8.00 & 0.5 & 1 & 1.208 & 3.608 & $3.639(35)$ & -0.9005 & 0.152 & 0.374 \\
\hline 6.00 & 0.5 & 1 & 1.292 & 3.594 & $3.616(24)$ & -0.9001 & 0.205 & 0.577 \\
\hline 4.00 & 0.5 & 1 & 1.489 & 3.553 & $3.582(23)$ & -0.8990 & 0.309 & 1.258 \\
\hline 3.00 & 0.5 & 1 & 1.735 & 3.487 & $3.521(24)$ & -0.8967 & 0.409 & 2.992 \\
\hline 2.50 & 0.5 & 1 & 1.989 & 3.401 & $3.441(21)$ & -0.8919 & 0.512 & 8.295 \\
\hline 2.30 & 0.5 & 1 & 2.237 & 3.315 & & -0.8789 & 0.688 & 23.680 \\
\hline 6.00 & 1.0 & 1 & 1.148 & 3.612 & $3.639(22)$ & -0.9006 & 0.112 & 0.247 \\
\hline 4.00 & 1.0 & 1 & 1.235 & 3.592 & $3.629(23)$ & -0.9001 & 0.171 & 0.426 \\
\hline 3.00 & 1.0 & 1 & 1.334 & 3.566 & $3.595(22)$ & -0.8995 & 0.233 & 0.666 \\
\hline 2.00 & 1.0 & 1 & 1.573 & 3.484 & $3.517(27)$ & -0.8971 & 0.360 & 1.498 \\
\hline 1.50 & 1.0 & 1 & 1.892 & 3.342 & $3.381(22)$ & -0.8917 & 0.503 & 3.629 \\
\hline 1.30 & 1.0 & 1 & 2.182 & 3.200 & & -0.8829 & 0.632 & 7.392 \\
\hline
\end{tabular}

Table 2

Effective field $W$, compressibility factor $\beta p / \rho$, and Fourier transforms at $k=0$ of three coefficients of the orthogonal polynomial expansion of the total correlation function $h=g-1$, for a biaxial nematic model at $\rho \sigma^{2}=0.8$

\begin{tabular}{|c|c|c|c|c|c|c|c|c|}
\hline \multirow[t]{2}{*}{$T^{*}$} & \multirow[t]{2}{*}{$\kappa$} & \multirow[t]{2}{*}{$W_{0}$} & \multirow[t]{2}{*}{$W$} & \multicolumn{2}{|l|}{$\beta p / \rho$} & \multirow[t]{2}{*}{$\rho \tilde{h}_{000}(0)$} & \multirow[t]{2}{*}{$\rho \tilde{h}_{220}(0)$} & \multirow[t]{2}{*}{$\rho \tilde{h}_{222}(0)$} \\
\hline & & & & RHNC & $\mathrm{MC}$ & & & \\
\hline 8.00 & 0.5 & 0 & 0.000 & 7.264 & $7.436(76)$ & -0.9674 & 0.415 & 0.415 \\
\hline 6.00 & 0.5 & 0 & 0.000 & 7.258 & $7.439(72)$ & -0.9674 & 0.644 & 0.644 \\
\hline 4.00 & 0.5 & 0 & 0.000 & 7.231 & 7.425 (79) & -0.9673 & 1.465 & 1.465 \\
\hline 3.50 & 0.5 & 0 & 0.000 & 7.210 & $7.380(73)$ & -0.9672 & 2.202 & 2.201 \\
\hline 3.20 & 0.5 & 0 & -0.001 & 7.186 & $7.368(81)$ & -0.9671 & 3.304 & 3.299 \\
\hline 3.10 & 0.5 & 0 & -0.002 & 7.171 & $7.369(82)$ & -0.9670 & 4.134 & 4.122 \\
\hline 6.00 & 1.0 & 0 & 0.000 & 7.260 & $7.434(42)$ & -0.9674 & 0.288 & 0.288 \\
\hline 4.00 & 1.0 & 0 & 0.000 & 7.244 & $7.435(40)$ & -0.9673 & 0.508 & 0.508 \\
\hline 3.00 & 1.0 & 0 & 0.000 & 7.218 & $7.395(37)$ & -0.9673 & 0.826 & 0.826 \\
\hline 2.00 & 1.0 & 0 & -0.003 & 7.086 & $7.315(32)$ & -0.9668 & 2.599 & 2.590 \\
\hline 1.95 & 1.0 & 0 & -0.005 & 7.057 & $7.307(38)$ & -0.9667 & 3.106 & 3.086 \\
\hline 1.93 & 1.0 & 0 & -0.007 & 7.042 & $7.286(45)$ & -0.9666 & 3.464 & 3.435 \\
\hline 8.00 & 0.5 & 1 & 1.304 & 7.227 & $7.407(45)$ & -0.9673 & 0.210 & 0.615 \\
\hline 6.00 & 0.5 & 1 & 1.436 & 7.202 & $7.399(32)$ & -0.9672 & 0.281 & 1.062 \\
\hline 4.00 & 0.5 & 1 & 1.769 & 7.113 & $7.304(41)$ & -0.9669 & 0.413 & 3.882 \\
\hline 3.40 & 0.5 & 1 & 2.048 & 6.993 & $7.218(47)$ & -0.9659 & 0.560 & 18.939 \\
\hline 6.00 & 1.0 & 1 & 1.221 & 7.232 & $7.425(45)$ & -0.9673 & 0.161 & 0.400 \\
\hline 4.00 & 1.0 & 1 & 1.362 & 7.195 & $7.387(50)$ & -0.9672 & 0.248 & 0.768 \\
\hline 3.00 & 1.0 & 1 & 1.530 & 7.136 & $7.326(51)$ & -0.9670 & 0.336 & 1.418 \\
\hline 2.50 & 1.0 & 1 & 1.692 & 7.066 & $7.276(30)$ & -0.9668 & 0.409 & 2.456 \\
\hline 2.10 & 1.0 & 1 & 1.938 & 6.924 & $7.161(26)$ & -0.9661 & 0.522 & 6.057 \\
\hline 2.01 & 1.0 & 1 & 2.096 & 6.822 & $7.130(59)$ & -0.9653 & 0.596 & 10.839 \\
\hline
\end{tabular}


external disorienting fields $W_{0}=1$ and 0 . The RHNC calculations used a range $R=20 \sigma$ with $N_{r}=1000$ grid points for the $\kappa=1.0$ cases and double these numbers for the longer-ranged $\kappa=0.5$ cases. The computer simulations have been carried out using a Monte Carlo (MC) method in the canonical ensemble with samples of 900 and 2025 particles. Thermodynamic and structural properties were calculated after $10^{4}$ equilibration moves followed by $10^{5}$ production moves for the ensemble averages. Each move implies $N$ translation and $N$ rotation trials ( $N$ being the number of particles). The isotropic-nematic transition was studied in the zerofield case using Saupe's tensor,

$Q_{a \beta}=\frac{1}{N} \sum_{i=1}^{N} \frac{1}{2}\left(3 s_{i \alpha} s_{i \beta}-\delta_{\alpha \beta}\right)$,

where $s_{i \alpha}$ is the $\alpha$ component of the unit vector describing the orientation of particle $i$. The eigenvector corresponding to the largest eigenvalue, $S$, determines the director $\hat{\mathbf{n}}$ of the nematic phase and $S$ is the order parameter describing the transition. In the presence of a finite field, $W_{0}>0$, the particles tend to arrange themselves perpendicular to the field, so that the order parameter $S$ is necessarily different from zero. Thus, $S$ is not a valid quantity to describe an in-plane transition. Therefore, we define a renormalized two-dimensional unit vector $\hat{\mathbf{u}}_{i}=\left(s_{i_{x}}, s_{i_{y}}\right) / \sqrt{s_{i_{x}}^{2}+s_{i_{y}}^{2}}$, which describes the orientation of particle $i$ within the plane. A corresponding two-dimensional Saupe's tensor is defined as

$Q_{\alpha \beta}^{2 d}=\frac{1}{N} \sum_{i=1}^{N} \frac{1}{2}\left(2 u_{i \alpha} u_{i \beta}-\delta_{\alpha \beta}\right)$,

where once more the order parameter, $S^{2 D}$, will be simply defined as the largest eigenvalue of $Q_{\alpha \beta}^{2 d}$.

Let us focus first on the thermodynamics. In Fig. 1, we have plotted the values of the excess internal energy for various temperatures and reduced densities, with and without external field. One observes particularly that for $\rho \sigma^{2}=0.6$ the RHNC results are in excellent agreement with simulation in all cases. For the higher reduced density of 0.8 , some deviations start to appear at low temperatures. The effective field $W$, pressure $p$, and three quantities $\rho \tilde{h}_{k l m}(0)$ which enter the isothermal compressibility and the susceptibility are presented in Tables 1 and 2. (The small negative values of $W$ for some low-temperature cases with $W_{0}=0$ are spurious effects of the present isotropic approximation for $b\left(r, \omega_{1}, \omega_{2}\right)$.)

To illustrate how the integral equation reproduces the orientational structure of the fluid, we have plotted in Fig. 2 the orientational order parameter $\left\langle P_{2}(\cos \theta)\right\rangle$ for the case $W_{0}=1$. (In the zero-field case, the values of this parameter are essentially zero for the temperatures

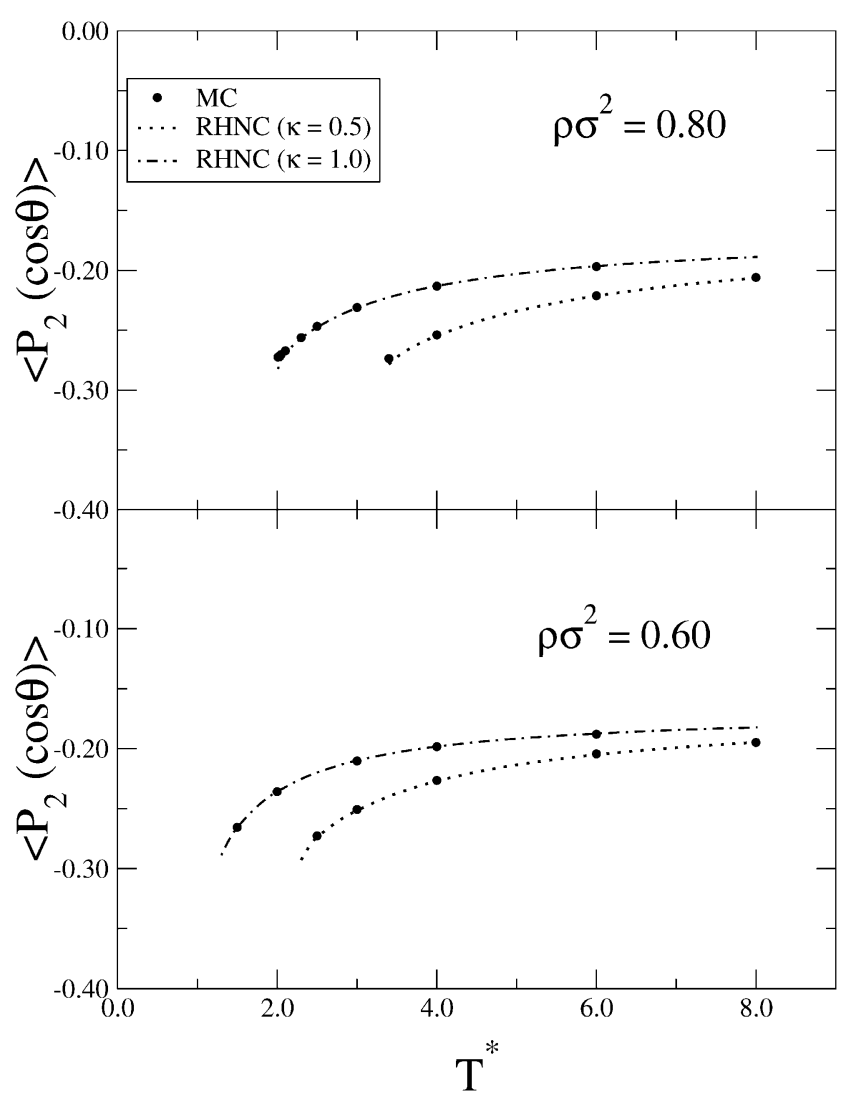

Fig. 2. Order parameter $\left\langle\frac{1}{2}\left(3 \cos ^{2} \theta-1\right)\right\rangle$ in the presence of a disorienting field for the biaxial nematic fluid in a plane.

at which the integral equation can be solved.) We note that this quantity is negative, indicating the tendency of the particles to orient themselves perpendicular to the field, i.e., to lie in the plane. This tendency is enhanced as the temperature is lowered and it is only slightly affected by the density; i.e., for the densities here considered, this quantity mostly reflects the individual response of the particles to the external field and is hardly a collective property. The agreement found for this order parameter can also be seen in the microscopic pair structure, whose most significant components are represented in Fig. 3. The behavior of the projection $\rho^{220}(r) / \rho^{2}$ indicates that the nematogens are organized mostly parallel to each other, and that the external field induces a long-range order so that this correlation no longer vanishes for large $r$. For the center-to-center distribution function $g_{000}(r)$ we have plotted just the results of one temperature and zero field, since for the present model the temperature and field dependence are negligible.

Turning now to the convergence properties of the integral equation, we find that convergence breaks down for reduced temperatures just below those reported in Tables 1 and 2 . From the behavior of $\rho \tilde{h}_{000}(0)$, one can 


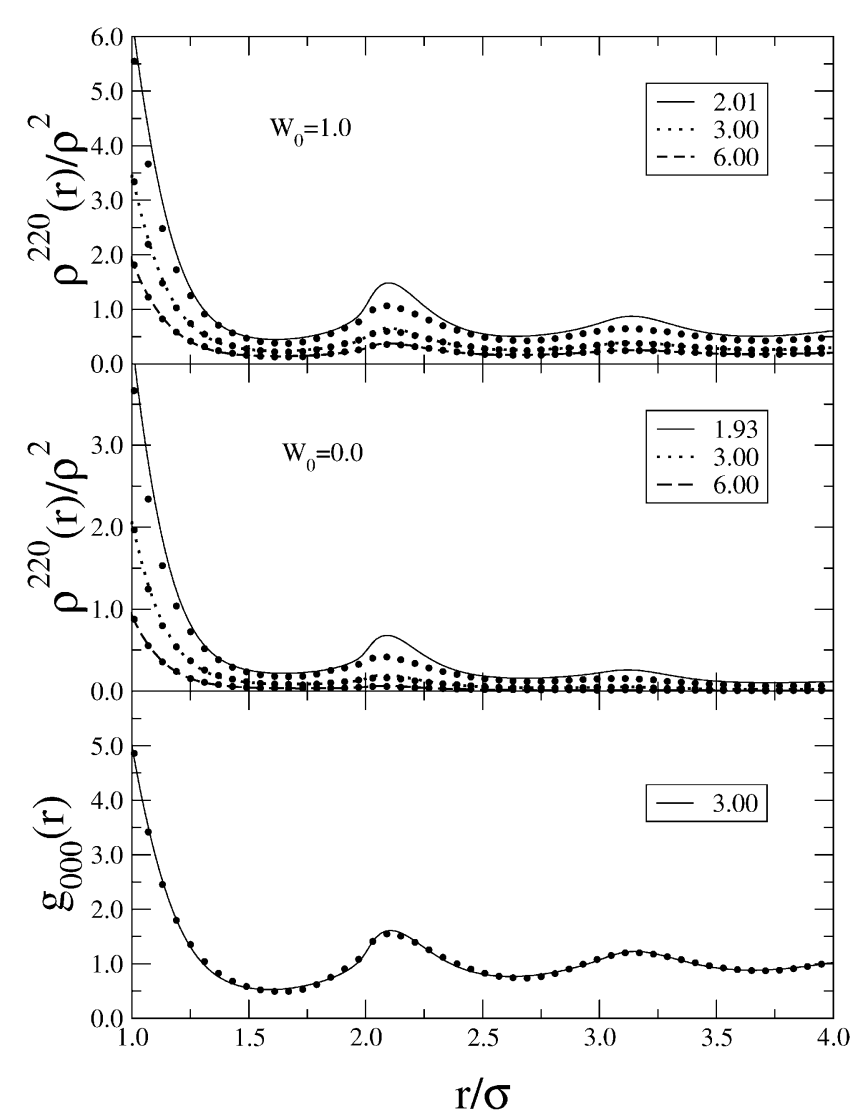

Fig. 3. Center-to-center distribution function $g_{000}(r)$ in zero field and angular projection $\rho^{220}(r) / \rho^{2}$ with and without a disorienting field, for the system of coplanar nematogens.

say that we are not in the vicinity of a condensation transition. However, one notes that the quantity $\rho \tilde{h}_{222}(0)$, which enters the $x y$-dependent elements of the susceptibility tensor, shows an evident increase before the integral equation solution breaks down. This points to the existence of an orientational order-disorder transition taking place at lower temperatures. In the zerofield case, $\rho \tilde{h}_{220}(0)$ - which is the leading component of the $z$-dependent elements of the susceptibility tensorexhibits the same behavior, with values very similar to those of $\rho \tilde{h}_{222}(0)$. This is consistent with a simple firstorder isotropic-nematic transition, with no preferred orientation of the director [22]. If one measures the response of the system to an external field in the direction of the $z$-axis, then the divergence in the $z$ components of the susceptibility simply signals the order-disorder transition, and the divergence of the $x y$ elements corresponds to Goldstone modes that reflect the negligible work needed to rotate a fully oriented phase at zero field in the thermodynamic limit. As the field is turned on, the transition is known to become second order and to appear at higher temperature, with the orientation of the nematogens lying basically in the plane [4]. Here we observe that $\rho \tilde{h}_{220}(0)$ grows as the temperature is lowered but never exceeds unity. In contrast, $\rho \tilde{h}_{222}(0)$ increases even more rapidly than in the zero-field case. This is clearly the signature of an order-disorder transition, which takes place essentially within the plane. In Tables 1 and 2 one can also see that the integral equation breaks down at a somewhat higher temperature in the presence of a disorienting field, as found in Ref. [4] for the OPR model.

With these theoretical results in mind, we carried out extensive MC simulations to analyze the behavior of the two- and three-dimensional order parameters, $S^{2 D}$ and $S$. For that purpose, we have considered sample sizes of 900 and 2025 particles for $\rho \sigma^{2}=0.8$ and $\kappa=1$, running simulations with $1 \times 10^{4}$ equilibration moves and $10^{5}$ production moves to obtain ensemble averages. The results of these calculations can be seen in Figs. 4 and 5 , respectively, without and with a disorienting field. We observe that in all cases the 2025 particle curves are steeper at the transition, as should be expected. In the zero-field case, both the three- and two-dimensional order parameters indicate a transition taking place at $T^{*} \approx 1.3$. When the disorienting field is turned on, the

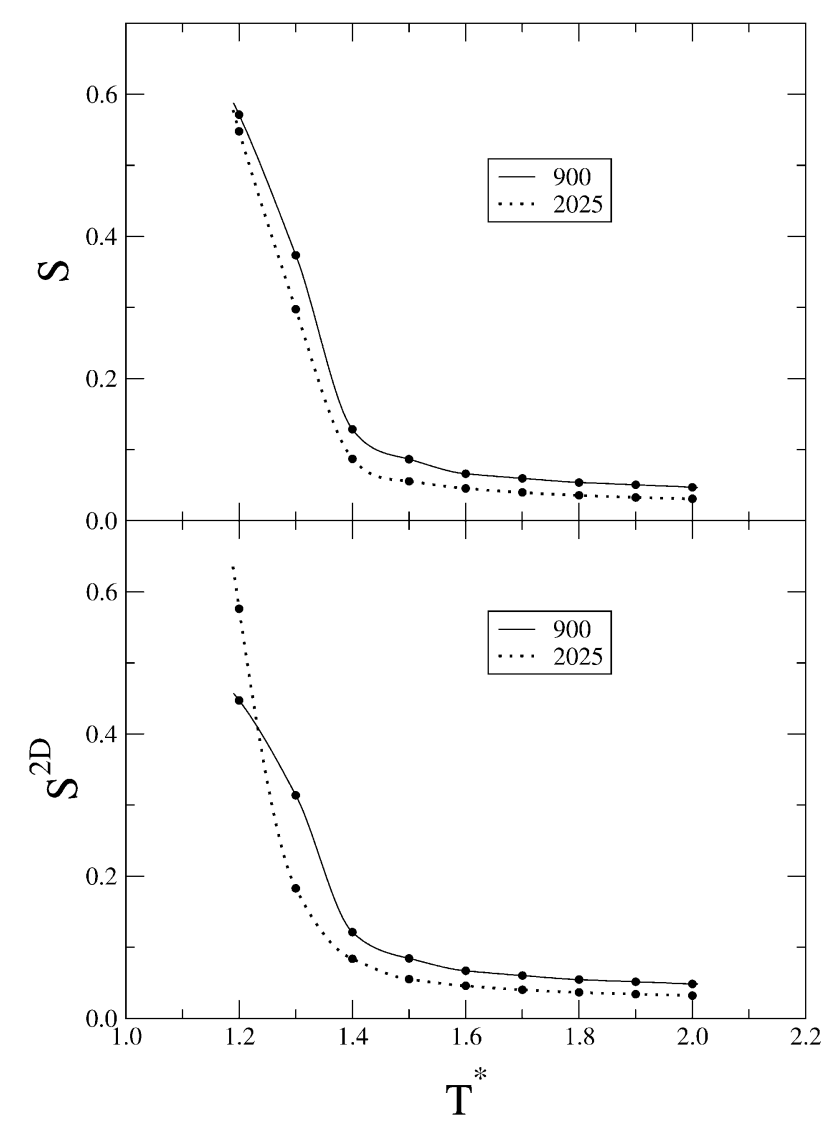

Fig. 4. Nematic order parameter in three dimensions, $S$, and on the plane, $S^{2 D}$, for the coplanar nematogens in the absence of disorienting field, from MC simulations. 


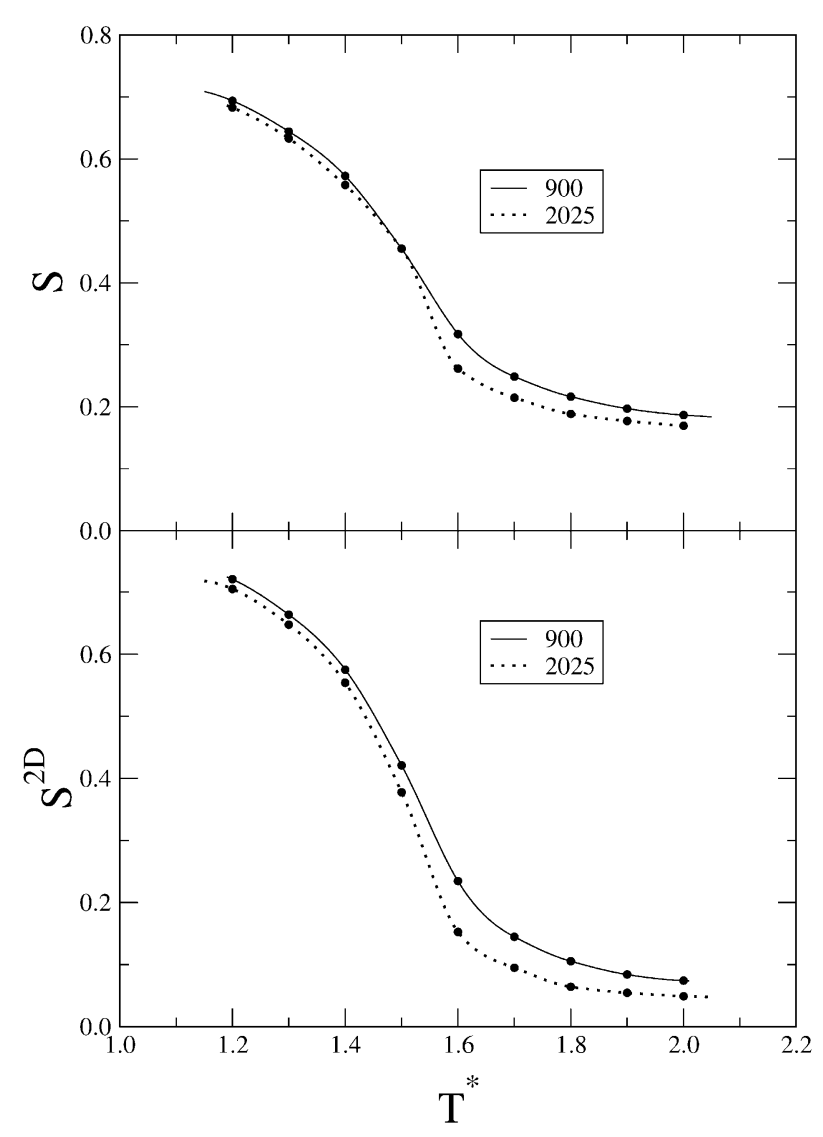

Fig. 5. Nematic order parameter in three dimensions, $S$, and on the plane, $S^{2 D}$, for the biaxial nematic fluid in a plane with disorienting field $W_{0}=1$, from MC simulations.

three-dimensional order parameter has non-negligible values for all temperatures. In contrast, the two-dimensional order parameter, $S^{2 D}$, varies from zero (or more properly $O(1 / \sqrt{N})$ ) to the values that are significantly positive. This is a clear indication that the nematogens are undergoing an orientational order-disorder transition in the $x y$ plane. The transition temperature can be estimated to lie around $T^{*}=1.5$, also slightly above the non-zero field case, as predicted by the RHNC results. A more detailed simulation study should include an analysis of the system-size dependence of Binder's cumulant and the critical parameters, so that a clear assessment of the order of the transition can be performed [23]. This will be the subject of future work.

In summary, we have presented the solution of the anisotropic $\mathrm{OZ}$ equation, in conjunction with the RHNC closure and the first member of the KBGY hierarchy, for a simple model of coplanar nematogens with and without a disorienting external field. The integral equation results in the non-oriented phase are in good agreement with computer simulation, although the theory overestimates the transition temperature, especially in the zero-field case. Perhaps this overestimation of the critical temperature is connected with the fact that the transition in the zero-field case is of the first order and that, while a similar integral equation is known to yield good critical temperature estimates for the second-order ferromagnetic transition of the Heisenberg spin fluid [10], the RHNC approximation considerably overestimates the critical temperature in the first-order gasliquid transition of simple Lennard-Jones fluids [24]. Whether this is a mere coincidence or a general trend for this type of integral equation remains to be further investigated.

\section{Acknowledgments}

E.L. and C.M. acknowledge support from the Dirección General de Investigación Científica y Técnica under Grant No. BFM2001-1017-C03-01.

\section{References}

[1] F. Egbert, L. Longa, W.H. de Jeu, Phys. Rep. 135 (1986) 195.

[2] S. Singh, Phys. Rep. 324 (2000) 107.

[3] C.P. Fan, M.J. Stephen, Phys. Rev. Lett. 25 (1970) 500.

[4] T.G. Sokolovska, R.O. Sokolovskii, M.F. Holovko, Phys. Rev. E 62 (2000) 6771.

[5] T.G. Sokolovska, R.O. Sokolovskii, M.F. Holovko, Phys. Rev. E 64 (2001) 051710.

[6] R.A. Lovett, C.Y. Mou, F.P. Buff, J. Chem. Phys. 65 (1976) 570.

[7] M. Wertheim, J. Chem. Phys. 65 (1976) 2377.

[8] T.L. Hill, Statistical Mechanics, McGraw-Hill, 1956, chap. 6.

[9] F. Lado, E. Lomba, Phys. Rev. Lett. 80 (1998) 3535.

[10] F. Lado, E. Lomba, J.J. Weis, Phys. Rev. E 58 (1998) 3478.

[11] E. Lomba, F. Lado, J.J. Weis, Phys. Rev. E 61 (2000) 3838.

[12] J.P. Hansen, I.R. McDonald, Theory of Simple Liquids, Academic Press, 1986.

[13] D. Henderson, in: D. Henderson (Ed.), Fundamentals of Inhomogeneous Fluids, Dekker, 1992, chap. 4.

[14] C.G. Gray, K.E. Gubbins, Theory of Molecular Fluids, Vol. 1, Oxford University Press, 1984.

[15] T.G. Sokolovska, Physica A 253 (1998) 459.

[16] A. Perera, Phys. Rev. E 60 (1999) 2912.

[17] S.H.L. Klapp, G.N. Patey, J. Chem. Phys. 112 (2000) 3832.

[18] F. Lado, Phys. Rev. A 8 (1973) 2548.

[19] F. Lado, J. Chem. Phys. 49 (1968) 3092.

[20] F. Lado, Comput. Phys. 8 (1971) 417.

[21] W.H. Press, S.A. Teukolsky, Comput. Phys. 4 (1990) 423.

[22] T.G. Sokolovska, M.F. Holovko, R.O. Sokolovskii, Ukr. Phys. J. 42 (1997) 1304.

[23] H. Weber, W. Paul, K. Binder, Phys. Rev. E 59 (1999) 2168.

[24] E. Lomba, Mol. Phys. 68 (1989) 87. 